\title{
Erratum to: Regulation of sporulation initiation by NprR and its signaling peptide NprRB: molecular recognition and conformational changes
}

\author{
Rosina Cabrera • Jorge Rocha • Víctor Flores • Luz Vázquez-Moreno • \\ Gabriel Guarneros • Gabriela Olmedo • Adela Rodríguez-Romero • \\ Mayra de la Torre
}

Published online: 15 October 2014

(C) Springer-Verlag Berlin Heidelberg 2014

\section{Erratum to:Appl Microbiol Biotechnol}

DOI 10.1007/s00253-014-6094-8

The original version of this article inadvertently contained an error.

In the discussion section we stated:

PapR peptide-binding to apo PlcR dimer promotes tetramerization; the tetramer is the active form of this transcriptional regulator (Grenha et al. 2012). However, this is incorrect; the results shown in the paper by Grenha et al. (2012) are in accord with the following statement:

PapR induces conformational changes in the PlcR dimer that allow proper positioning of the DNA binding domain on its DNA recognition sequence (Grenha et al. 2012).

The online version of the original article can be found at http:// dx.doi.org/10.1007/s00253-014-6094-8.

R. Cabrera $\cdot$ J. Rocha $\cdot$ L. Vázquez-Moreno $\cdot$ M. de la Torre $(\triangle)$ Centro de Investigación en Alimentación y Desarrollo A. C., Km 0.6 Carretera a La Victoria, 83304 Hermosillo, Sonora, Mexico e-mail: mdelatorre@ciad.mx

V. Flores $\cdot$ G. Guarneros

Departamento de Genética y Biología Molecular, Centro de Investigación y de Estudios Avanzados del Instituto Politécnico Nacional, Av. Instituto Politécnico Nacional 2508 Col. San Pedro Zacatenco, 07000 Mexico, Distrito Federal, Mexico

G. Olmedo

Departamento de Ingeniería Genética, Centro de Investigación y de Estudios Avanzados del Instituto Politécnico Nacional, Unidad Irapuato, Km. 9.6 Libramiento Norte Carretera Irapuato-León, 36821 Irapuato, Guanajuato, Mexico

\section{A. Rodríguez-Romero}

Instituto de Química, Universidad Nacional Autónoma de México, Circuito Exterior, Ciudad Universitaria, 04510 Mexico, Distrito Federal, Mexico 